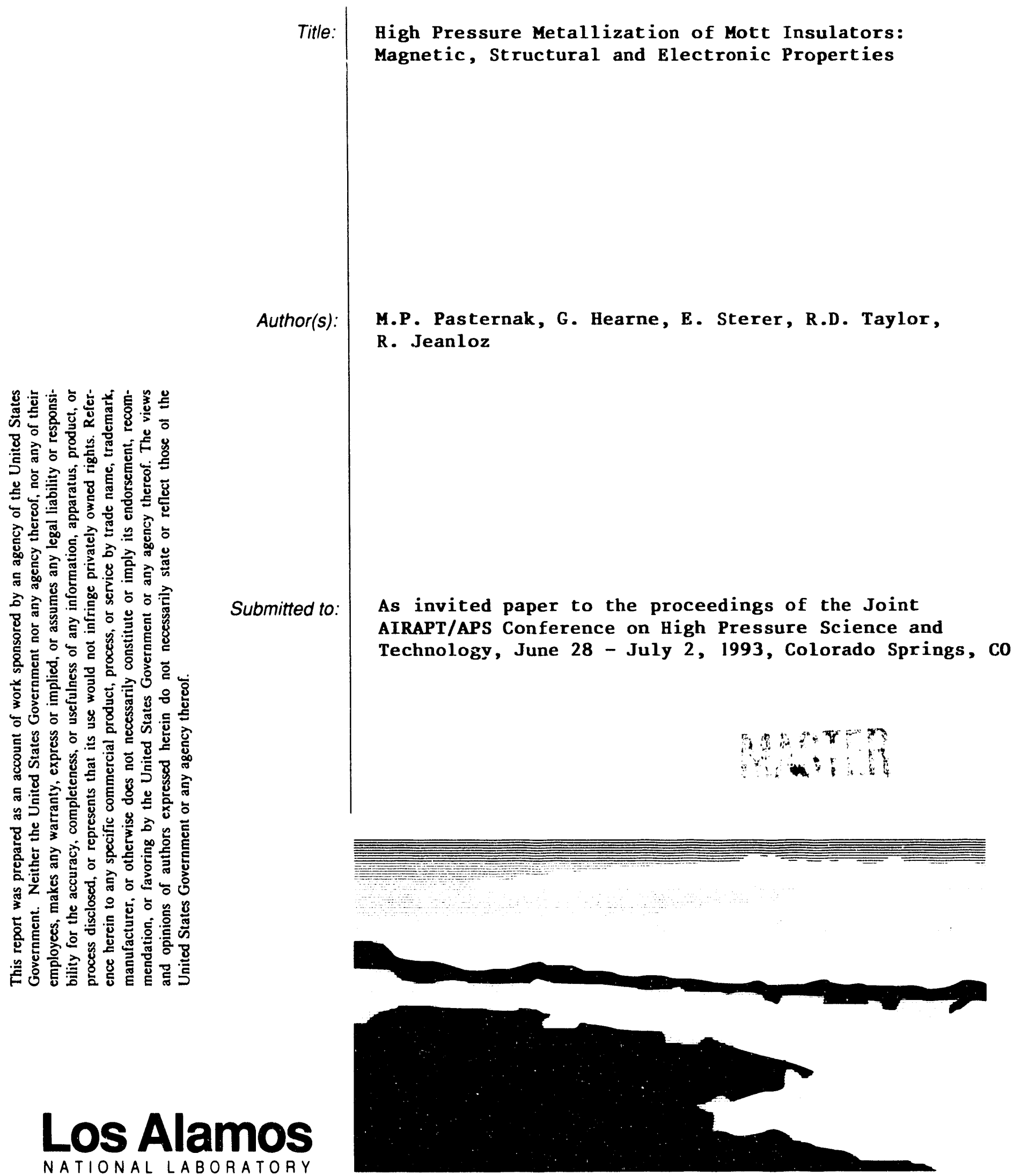

Los Alamos National Laboratory, an aftirmative action/equal opportunity emplóyer, is operated by the University of California for the U.S. Department of Energy under contract W-7405-ENG-36. By acceptance of this article, the publisher recognizes that the U.S. Government retains a nonexclusive, royalty-free license to publish or reproduce the published form of this contribution, or to allow others to do so, for U.S. Government purposes. The Los Alamos National Laboratory requests that the publisher identify this article as work performed under the auspices of the U.S. Department of Energy. 


\section{HIGH PRESSURE METALLIZATION OF MOTT INSULATORS; MAGNETIC, STRUCTURAL AND ELECTRONIC PROPERTIES}

M. P. Pasternak, Tel Aviv University

G. Hearne, Tel Aviv University

E. Sterer, Tel Aviv University

R.D. Taylor, P-10, LANL

R. Jeanloz, UC Berkeley

Invited full paper for publication in High Pressure Research

Submitted July 20, 1993

LA-UR-93-

The discovery of high-temperature superconductors in doped $\mathrm{Cu}$-oxides has renewed the interest in one of the oldest fundamental (and still unsolved problems) in condensed matter physics, namely, the metal-insulator transition in the so called Mott Insulators (MI). Most of $3 \mathrm{~d}$ transition-metal (TM) compounds, (particularly the binary ones) are MI, characterized by very narrow $3 \mathrm{~d}$ bands where electron-electron interaction is strong. Thus the electron correlation effects are predominant, leading to the breakdown of the simplist: $=$ one-electron band theory.

Metallic conductivity in MI can be achieved in two ways, namely, via valence changes and band-gap closure. In the first procedure the mean valence of the TM ions are shifted from an integral to intermediate values by chemical substitution or stoichiometry control. This leads to doping of extra electron or hole carriers. However, in contrast to classical semiconductors, chemical substitution of narrow band insulators also results in severe electronic and structural disorder in the MI under study. The second procedure, the band-gap closure, is achieved by reducing the splitting between energy bands and/or increasing the bandwidth. Due to relatively large band gap energies characteristic to most MI, this is possible only by applying external pressure.

In this article we address the pressure-induced Mott Transition (MT) into a metallic state by the gap-closure mechanism. The band gap of the MI can be classified into two main types: (i) the d-d Mott-Hubbard ${ }^{3}$ (intra-band gap) involving the $d-d$ Coulomb repulsive energy $U$, or (ii) of the ligand-p to metal-d charge-transfer (CT) type (inter-band gap). The materials studied were representative of the structurally isomorphous (TM) $\mathrm{I}_{2}(\mathrm{TM}=\mathrm{V}, \mathrm{Fe}, \mathrm{Co}$ and $\mathrm{Ni}$ ) compounds. They consist of alternating layers of weakly interacting I-TM-I slabs and are antiferromagnetic with $\mathrm{T}_{\mathrm{N}}$ of $15,10,12$ 
and $75 \mathrm{~K}$ corresponding to $\mathrm{V}, \mathrm{Fe}, \mathrm{Co}$ and $\mathrm{Ni}$, respectively. The TM cation sublattice moments are coupled ferromagnetically.

As suggested by Zaanen et al. [Phys. Rev. Lett. 55, 418 (1985)], VI2 represents a typical case of a pure Mott-Hubbard type insulators whereas the $\mathrm{Fe}, \mathrm{Co}$, Ni diiodes are of the CT type. High pressure studies of the electronic, magnetic and structural properties of the (TM) $\mathrm{I}_{2}$ were carried out in the insulator and the metallic pressure ranges. The experimental tools used were: Resistivity, ${ }^{57} \mathrm{Fe}$ and ${ }^{129}$ I Mossbauer Spectroscopy (MS), and X-ray diffraction (XRD) using diamond anvil cells (DAC) to provide unique information for characterizing the magnetic and electronic properties of the $\mathrm{MI}$ and the nature of the MT. It was shown that, (i) the pressure at which the insulator-metal transitions occur $(\mathrm{Pc})$ coincides precisely with that of the loss of magnetism and (ii) the dbands remain extremely narrow up to the highest pressure applied. 


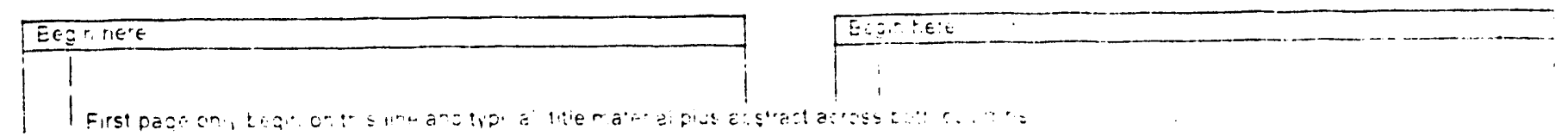

\title{
HIGH PRESSURE METALLIZATION OF MOTT INSULATORS; MAGNETIC, STRUCTURAL AND ELECTRONIC PROPERTIES*
}

\author{
Moshe P. Pasternak, Giovanni Hearne and Eran Sterer \\ School of Physics and Astronomy, Tel Aviv University, 69978, ISRAEL \\ R.Dean Taylor \\ Physics Division, Los Alamos National Laboratory, Los Alamos, NM 87545 \\ and \\ Raymond Jeanloz \\ Department of Geology and Geophysics, University of California
}

High pressure studies of the insulator-metal transition in the $(T M) I_{2}(T M=V, F e$, Co and $\mathrm{Ni}$ ) compounds are described. Those divalent transition-metal iodides are structurally isomorphous and classified as Mott Insulators. Resistivity, X-ray diffraction and Mössbauer Spectroscopy were employed to investigate the electronic, structural, and magnetic properties as a function of pressure both on the highly correlated and on the metallic regimes.

\section{INTRODUCTION}

The discovery of high-temperature superconductors in doped Cu-oxides renewed the interest in one of the oldest fundamental and still unsolved problems in condensed matter physics, namely, the metal-insulator transition in the so called Mott Insulators (MI) ${ }^{1}$. Most of $3 d$ transition-metal (TM) compounds, (particularly the binary ones) are MI, characterized by very narrow $3 d$ bands where electron-electron interaction is strong. Thus the electron correlation effects are predominant, leading to the breakdown of the simplistic one-electron band theory.

Metallic conductivity in MI can be achieved in two ways, namely, via valence changes and band-gap closure. In the first procedure the mean valence of the TM ions are shifted from an integral to intermediate values by chemical substitution or stoichiometry control. This leads to doping of extra electron or hole carriers. However, in contrast to ies to bottom classical serniconductors, chemical substitution of ngie spacir.g narrow band insulators also results in severe electronic and structural disorder in the MI under study. The second procedure, the band-gap closure, is achieved by reducing the splitting between energy bands and/or increasing the bandwidth. Due to relatively large band gap energies characteristic to most MI, this is possible only by applying external pressure?

In this article we address the pressure-induced Mott transition (MT) into a metallic state by the gap-closure mechanism. The band gap of the $\mathrm{Ml}$ can be classified into two main types: (i) the $d$-d MottHubbard $^{3}$ (intra band gap) involving the $d-d$ Coulomb repulsive energy $U$ or (ii) of the ligand-p to metal- $d$ charge-transfer (CT) type (inter band gap), involving the $d_{1} \rightarrow d_{1}^{n+1} L$ energy $\Delta$, where $L$ denotes a hole in the anion valence band ${ }^{4}$. The materials studied were representative of the structurally isomorphous (TM) $I_{2}$ (TM $=\mathrm{V}, \mathrm{Fe}, \mathrm{Co}$ and $\mathrm{Ni}$ ) compounds with their closely related space groups $R 3 \mathrm{~m}$ or $\mathrm{C} 3 \mathrm{~m}$. They consist of alternating layers of weakly interacting I-TM-I slabs and are antiferromagnetic with $T_{N}$ of 15, 10, 12 and $75 \mathrm{~K}$ corresponding to $\mathrm{V}, \mathrm{Fe}, \mathrm{Co}$ and $\mathrm{Ni}$, respectively. The TM cation sublattice moments are coupled ferromagnetically.

As suggested by Zaanen et al.(Ref. 4), $\mathrm{VI}_{2}$ represents a typical case of a pure Mott-Hubbard type in- 
BEgंn $\epsilon^{\prime} \epsilon$

sulators whereas the $\mathrm{Fe}, \mathrm{Co}, \mathrm{Ni}$ diiodides are of the CT type. High pressure studies of the electronic, magnetic and structural properties of the $(T M) I_{2}$ were carried out in the insulator and the metallic pressure ranges. The experimental tools used were: Resistivity, ${ }^{57} \mathrm{Fe}$ and ${ }^{129} \mathrm{I}$ Mössbauer Spectroscopy (MS), and X-ray diffraction (XRD) using diamond anvil cells (DAC).

\section{EXPERIMENTAL}

\section{Samples Preparation}

Samples were synthesized from the direct solidvapor reaction of the elements taking place in an evacuated quartz tube heated to $700^{\circ} \mathrm{C}$. The MS samples were prepared from enriched isotopes of ${ }^{57} \mathrm{Fe}$ and 129. All the $(T M) I_{2}$ are highly hygroscopic and special precautions were taken when loading into the DAC's.

\section{Measurements}

The DAC's used in the present experiments were the Merrill-Bassett triangular-type ${ }^{5}$ or related miniature-type ${ }^{6}$. The ruby fluorescence method 5 was used for manometry. The pressure and temperature ranges covered were 0 - $50 \mathrm{GPa}$ and 4 - $350 \mathrm{~K}$ respectively.

Resistivity measurements were performed using the quasi - four point method with $10-\mu \mathrm{m}$ thick gold electrodes. At pressures close to the metal-insulator transition $\left(\mathrm{P}_{\mathrm{c}}\right)$ the temperature dependence of $R(P)$ was obtained. $P_{c}$ was determined as the pressure at which the slope of $R(T)$ became positive, namely the material became metallic.

Mössbauer Spectroscopy was carried out using the $27.8-\mathrm{keV}{ }^{129}$ I resonance in all (TM) $I_{2}$ and the $14.4-$ $\mathrm{keV}{ }^{57} \mathrm{Fe}$ resonance in $\mathrm{FeI}_{2}$. A $\mathrm{Ta}_{0.90} \mathrm{~W}_{0.10}$ gasket/collimator was used and samples diammeters were es to botrom in the $300-800-\mu \mathrm{m}$ range. A detailed description of iglespac:n the ${ }^{129} \mathrm{I}$ MS is found in Ref. 7. For the ${ }^{57} \mathrm{FeI}_{2} \mathrm{MS}$ if footrotes measurements a commercial $5-\mathrm{mCi}^{57} \mathrm{Co}(\mathrm{Rh})$ point source of $0.5 \times 0.5 \mathrm{~mm}$ dimension was used ${ }^{8}$. At each pressure data were collected as a function of temperature from which we determine the pressure dependence of $T_{N}$, the hyperfine field $\mathrm{H}$, Quadrupole Splitting QS and Isomer Shift IS. At $T>T_{N}$ the Mössbauer spectra were determined solely by the quadrupole interaction whereas at $T<T_{N}$ by the combined magnetic and quadrupole interactions. For a polycrystalline sample with randomly oriented crystallographic and magnetic axes with respect to the direction of the $r$-ray the Spin-Hamiltonian $-H$ - governing the hyperfine interaction can be expressed as:

$$
\begin{gathered}
H=\mu H\left(I_{z} / I\right)+ \\
\left\{e^{2} q_{z z} Q\left(3 \cos ^{2} \theta-1\right) /[8 I(2 I-1)]\right\}\left\{3 I_{z}^{2}-I(1-1)\right\}
\end{gathered}
$$

where $\mu$ is the nuclear moment, $I_{z}$ and $l_{z}^{2}$ are spin operators and $e_{z z}$ is the principal axis of the electric-field-gradient ( $f f g$ ). In the $(\mathrm{TM}) \mathrm{I}_{2}$ the $\mathrm{ff}$ along the c-axis and is axially symmetric. For each spectrum the MS parameters, namely, $H, e^{2} q_{z 2} Q, \theta$ and the IS were determined from the experimental points using an approyriate least-squares fitting program. At each pressure the temperature depeudence of $\mathrm{H}$ allows the measurement of $T_{N}$, the temperature at which $\mathrm{H} \rightarrow \mathbf{0}$.

XRD patterns were collected at $300 \mathrm{~K}$ using a Guinier spectrometer. Main emphasis was at pressures near $P_{c}$ in order to detect and characterize possible crystallographic phase transitions. In all cases no structural changes were observed at the insulatormetal pressure transition.

\section{RESULTS and DISCUSSION}

\section{Magnetism in the Mott Insulator Phase.}

Any magnetic insulator, a material whose local moments and energy gap persist above the spin ordering temperature, can be classified as a Mott insulator, and vice versa. As long as the $3 d$-bands are well localized one would expect an increase in $T_{N}$ and no variation in the local moments with increasing pressure. The first experimental proof for this statement 
was provided by Pasternak et al. in the case of $\mathrm{NiI}_{2}$ (Ref. 9).

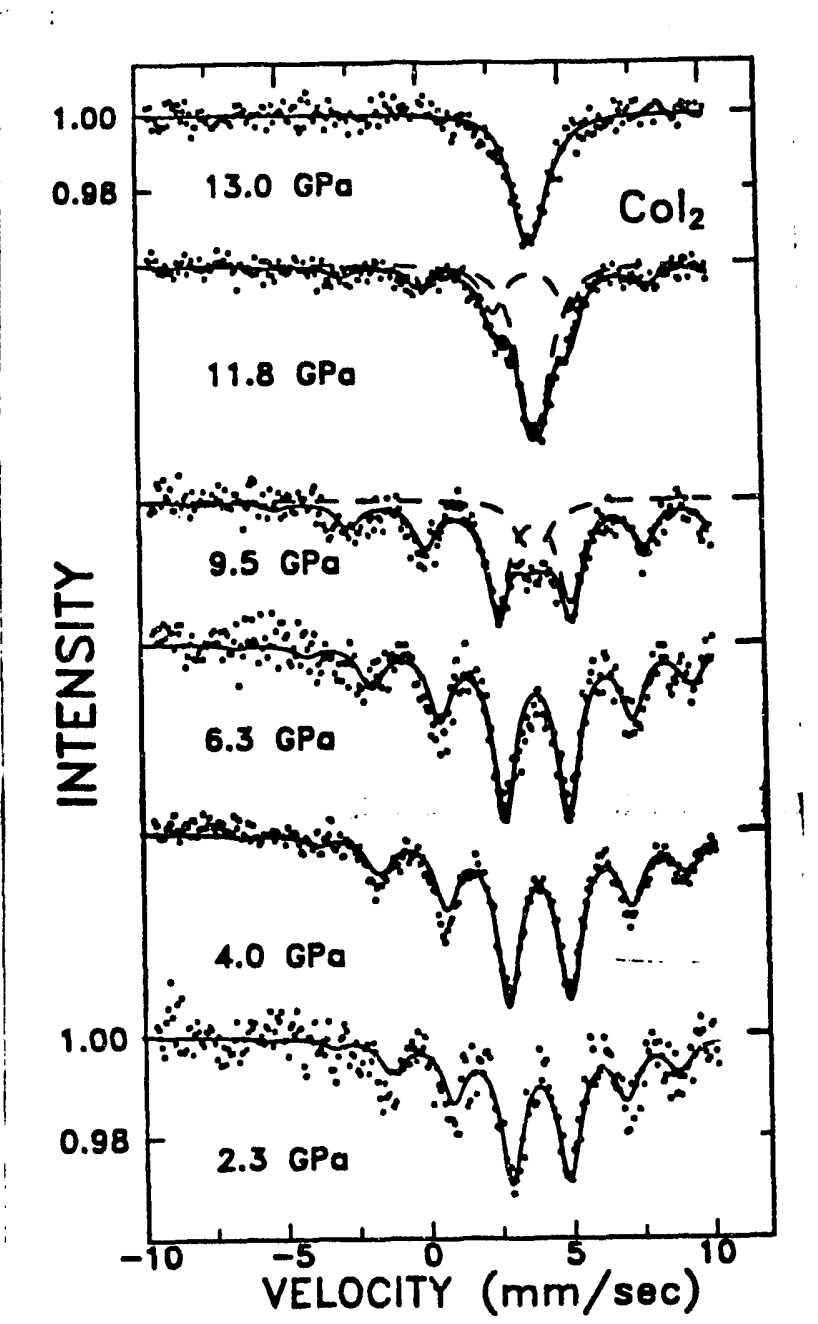

Fig.1 Typical Mössbauer spectra of $\mathrm{CoI}_{2}$ at $4 \mathrm{~K}$ recorded at several pressures. The solio iine is a theoretical curve obtained by least-squared fitting to the experimental points. The source is $\mathrm{Mg}_{3}{ }^{129 m} \mathrm{TeO}_{6}$.

The pressure variation of the 1291 hyperfine field $\mathrm{H}$ in $\mathrm{CoI}_{2}$ at $4 \mathrm{~K}\left(\mathrm{~T} \propto \mathrm{T}_{\mathrm{N}}\right)$ is shown in Fig. 1. The hyperfine field at low $T$ is proportional to the $\mathrm{Co}^{2+}$ moment. As can be seen, the field at $13 \mathrm{GPa}$ has collapsed. Within the $0-13 \mathrm{GPa}$ pressure range the stioberse geterencis MI regime persists. The variation in pressure of $T_{N}$, i focinc:ss, $H$ and IS is shown in Fig. 2. New data on $H(P)$ and $\mathrm{T}_{\mathrm{H}}(\mathrm{P})$ as measured with ${ }^{57} \mathrm{Fe}$ in $\mathrm{FeI}_{2}$ are shown in Fig. 3.

\section{The Mott Transition.}

The concurrence of an isostructural insulatormetal transition and of $d$-electron delocalization are inherent features of a MT. The insulator-metal transition is uniquely determined by resistivity measurements ${ }^{10}$. The pressure variation of $\log (R)$ for $\mathrm{CoI}_{2}$ is shown in Fig. 3. One clearly sees the dramatic decrease of $R$ with increasing $P$ (by seven orders of magnitude) leveling off at $P>12 \mathrm{GPa}$. As mentioned before, $P_{c}$ is determined from $R(T)$ measurements.

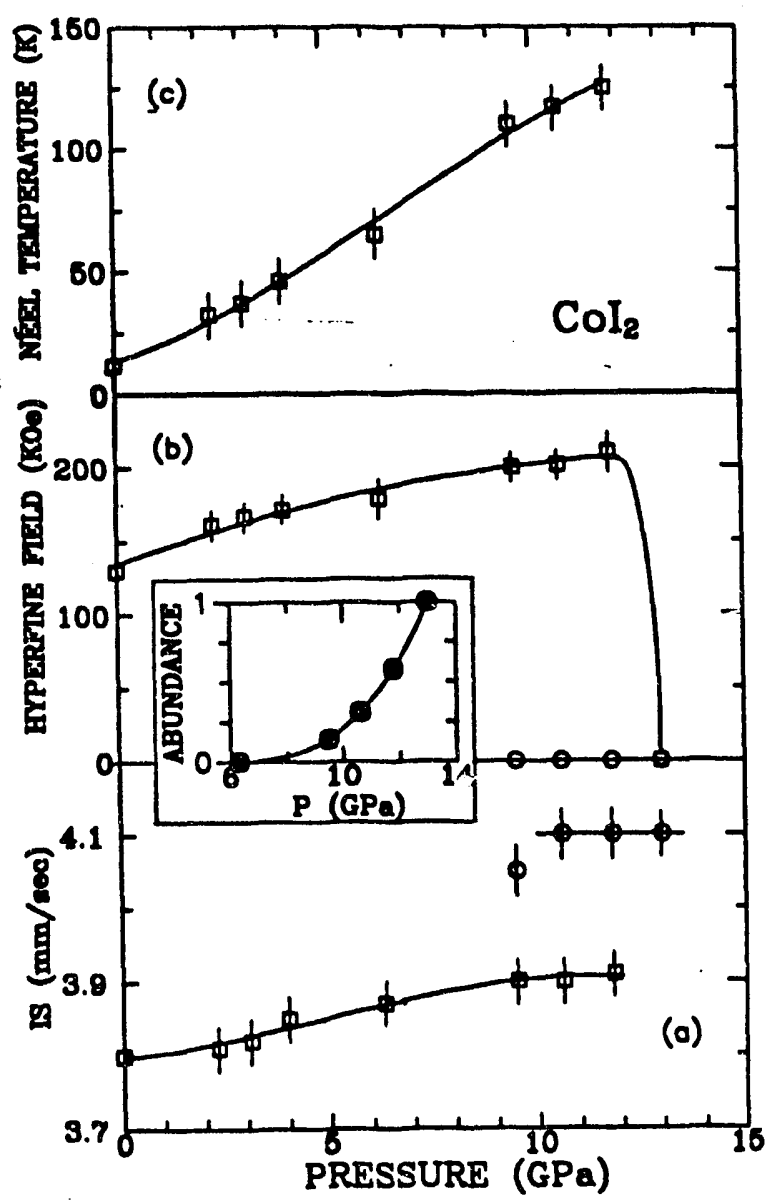

Fig. 2 Pressure dependence of the IS (a), $H$ (b) and $\mathrm{T}_{\mathrm{N}}$ (c) of $\mathrm{Col}_{2}$. The insert shows the pressure dependence of the abundance of the metallic state. Note the jump in IS due to the MT.

The effect of gap band closure, the MT, on the magnetic properties of the $\mathrm{MI}$ is to effectively cancel the local moments. This is indeed observed in the MS studies. The case of $\mathrm{CoI}_{2}$ is quite unique 


\section{Es:an no:}

unlike $\mathrm{NiI}_{2}$. Within $7<\mathrm{P}<13 \mathrm{GPa}$ one observes the coexistence of insulating (correlated) and metallic states; the abundance of the latter increases with increasing $P$, reaching unity at $\approx 13 \mathrm{GPa}$ (insert in Fig. 2). This phenomena is not detected by the Resistivity measurements. Within this pressure range the $R(T)$ slope is positive indicating a metallic regime and yet $R(\mathrm{P})$ decreases in value! The nature of the metallic behavior is due to the percolative mechanism. A complete transition into the nonmagnetic state is rather sharp, providing a well defined and more reliable method for determining $\mathbf{P}_{c}$. Values of $P_{c}$ for the $V, C o$ and $\mathrm{Ni}$ are 44, 13 and 19 $\mathrm{GPa}$, respectively. $\mathrm{P}_{c}$ of $\mathrm{FeI}_{2}$ has not yet been determined, but it is greater than $29 \mathrm{GPa}$.

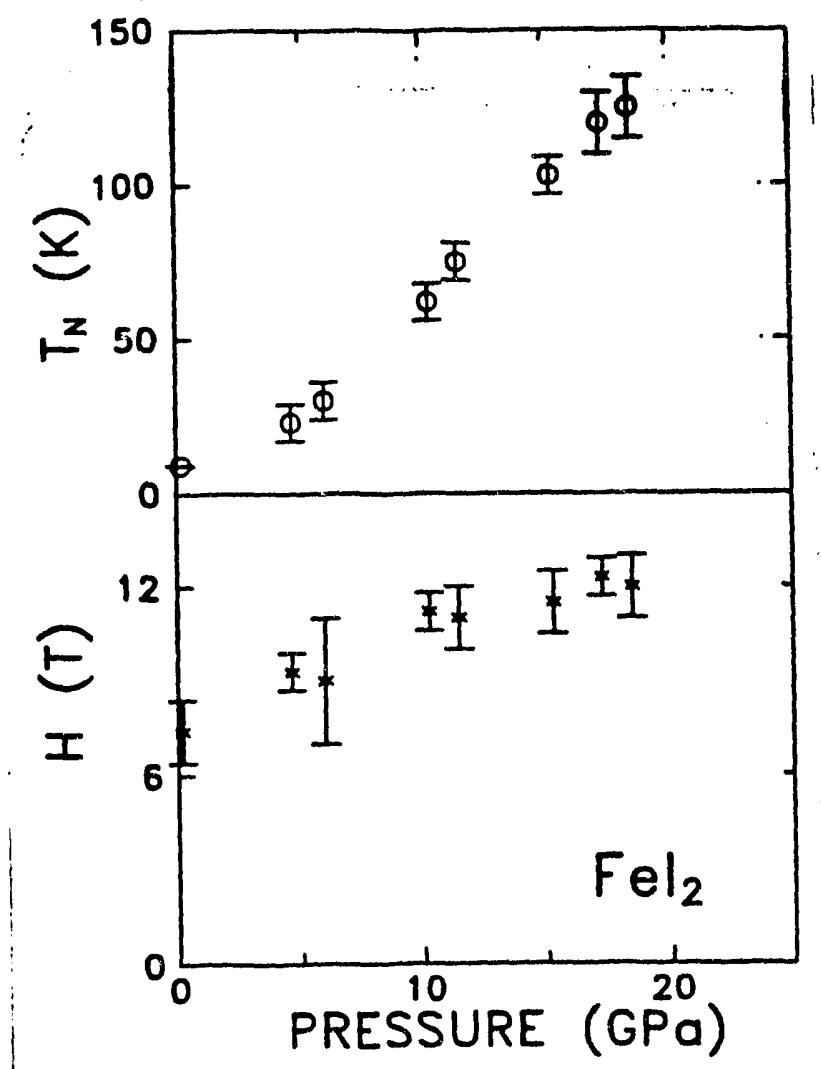

Fig.3 The pressure dependence of $\mathrm{H}$ and $\mathrm{T}_{\mathrm{N}}$ in ${ }^{57} \mathrm{FeI}_{2}{ }^{\text {* }}$ in the pressure range of $0-19 \mathrm{GPa}$.

According to Zaanen et al. ${ }^{4}$ the CT mechanism dominates the "heavy" transition metal compounds.

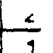

However there is no way a priori to predict the nature of the gap-closure at those high pressures. The ${ }^{229}$ MS provides an unique tool through the IS for assessing the nature of the MT. The inter-band closure which causes the $I^{-} 5 p$-band population to decrease, concomitantly transferring electrons into the empty $3 d$ band; this will directly affect the IS. It has been shown that the ${ }^{129} 1$ IS behaves linearly ${ }^{11}$ with the number of $5 p$-holes $\left(h_{p}\right)$ (with respect to a $\mathrm{Mg}_{3} \mathrm{TeO}_{6}$ source) $)^{12}$ :

$$
\text { IS }=1.27 h_{p}+3.6(\mathrm{~mm} / \mathrm{s})
$$

In case of $\mathrm{NiI}_{2}$ (Ref. 9) no abrupt change is observed in IS(P) at and around $P_{c}$, whereas the IS of metallic $\mathrm{CoI}_{2}$ increases abruptly by $0.20 \mathrm{~mm} / \mathrm{s}$ (see Fig. 4) corresponding to $0.16 h_{p}$ per ligand. Thus according to the ${ }^{129} \mathrm{I}$ MS data the $\mathrm{NiI}_{2} \mathrm{MT}$ is of a Mott-Hubbard type (intra-band) whereas that of $\mathrm{CoI}_{2}$ is of the CT (inter-band). Experiments are now underway to determine the Mott character of $\mathrm{VI}_{2}, \mathrm{MnI}_{2}$ and $\mathrm{FeI}_{2}$.

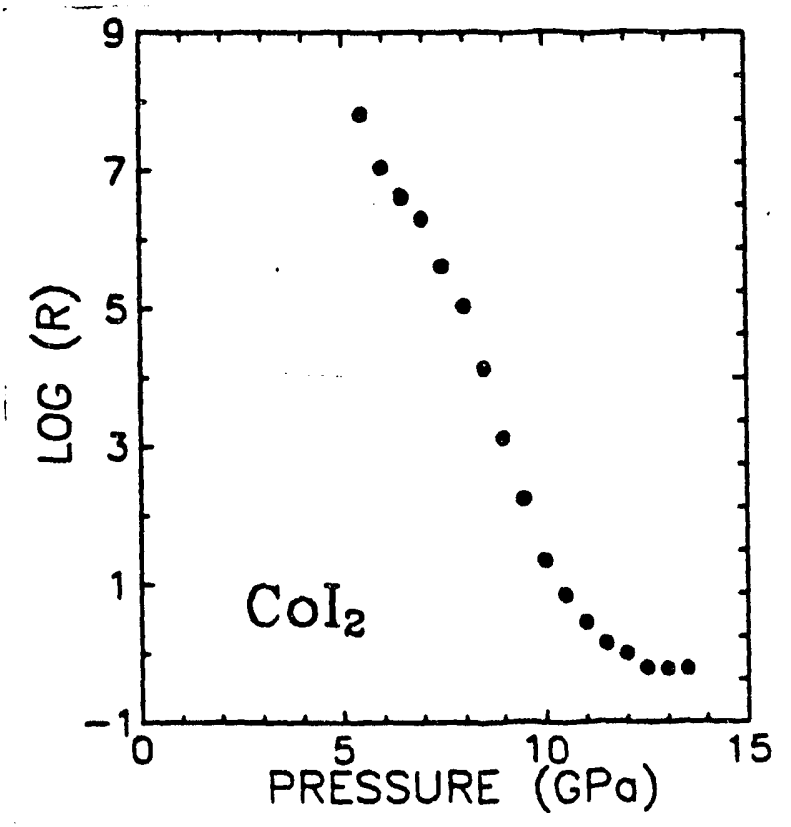

Fig. 4 Pressure dependence of the Resistance of $\mathrm{CoI}_{2}$ measured at $300 \mathrm{~K}$. The curve levels off near $P_{c}$. 


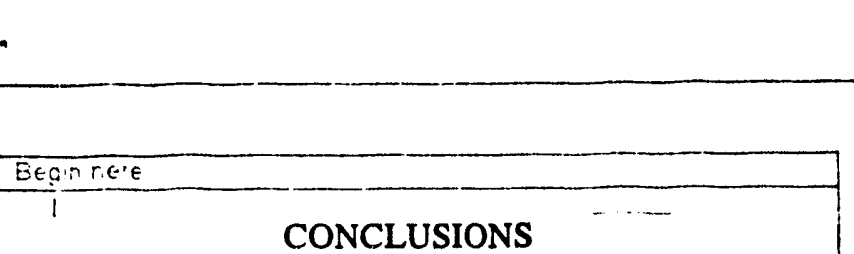

The combined XRD, MS and Resistivity methods used in conjunction with DACs have been shown to provide unique information for characterizing the magnetic and electronic properties of the $\mathrm{MI}$ and the nature of the MT. It was shown that, (i) the pressure at which the insulator-metal transitions occur $\left(\mathrm{P}_{\mathrm{c}}\right)$ coincides precisely with that of the loss of magnetism and (ii) the $d$-bands remain extremely narrow up to the highest pressure applied. This last observation is in accordance to the recent findings ${ }^{2}$ in the $\mathrm{RNiO}_{3}$ where CT transitions were observed at ambient pressure with change of temperature alone.

\section{REFERENCES}

* Supported in part by grants from the DOE-IGPP and the Israeli Basic Research Foundation.

1 - N.F Mott, Proc. Phys. Soc. London, A62, 416, 1949; G.A. Sawatzky, in Earlier and Recent Aspects of Superconductivity, Eds..G. Bednorz and K.A. Müller, Heidelberg: Springer-Verlag Berlin pp. 345-376, 1990; N. Tsuda, K. Nasu, A. Yanase and K. Siratori, Electronic Conduction in Solids, Springer-Verlag, Berlin, 1991.

2 - An exceptional case where gap closure was obtained by temperature increase at ambient pressure are some of the rare-earth nickelate perovskites, see J.B. Torrance et al. Phys Rev. B45, 8209, 1992.

3 - J. Hubbard, Proc. Roy. Soc. London A277, 237, 1964; ibid. A281, 401, 1964.

4 - J. Zaanen, G.A. Sawatzky and J.W. Allen, Phys. Rev. Lett. 55, 418, 1985.

5 - A. Jayaraman, Rev. Mod. Phys. 55, 65, 1983.

6 - E. Sterer, M.P. Pasternak and R.D. Taylor, Rev. Sci. Instrum. 71, 1117, 1990.

s io botior 7 - M.P. Pasternak and R.D. Taylor, Hyperfine jle spacn: Interact. 47, 415, 1989; ibid. 53, 159, 1990.

- fostrcies 8 - G.R. Hearne, M.P. Pasternak and R.D. Taylor, Hyperfine Interact., to be published.
Estricet

9 - M.P. Pasternak, R.D. Taylor, A. Chen, C. Meade, L.M. Falicov, A. Giesekus, R. Jeanloz and P.Y. Yu, Phys. Rev. Lett. 65, 790, 1990.

10 - The gap closure of the CT-type MI can in principle be determined by optical spectroscopy. However, the evolution of low energy gap semiconductors at pressures below $P_{c}$ may be misleading, inferring a metallic state to an actual gapped state.

11 - S.L. Ruby and G.K. Shenoy, Mössbauer Isomer Shifts, G.K. Shenoy and F.E. Wagner eds., Amsterdam: North Holland, 1978, p 617.

12 - M. Van der Heyden, M.P. Pasternak and G. Langouche, J. Phys. Chem. Solids 46, 1221, 1985. 

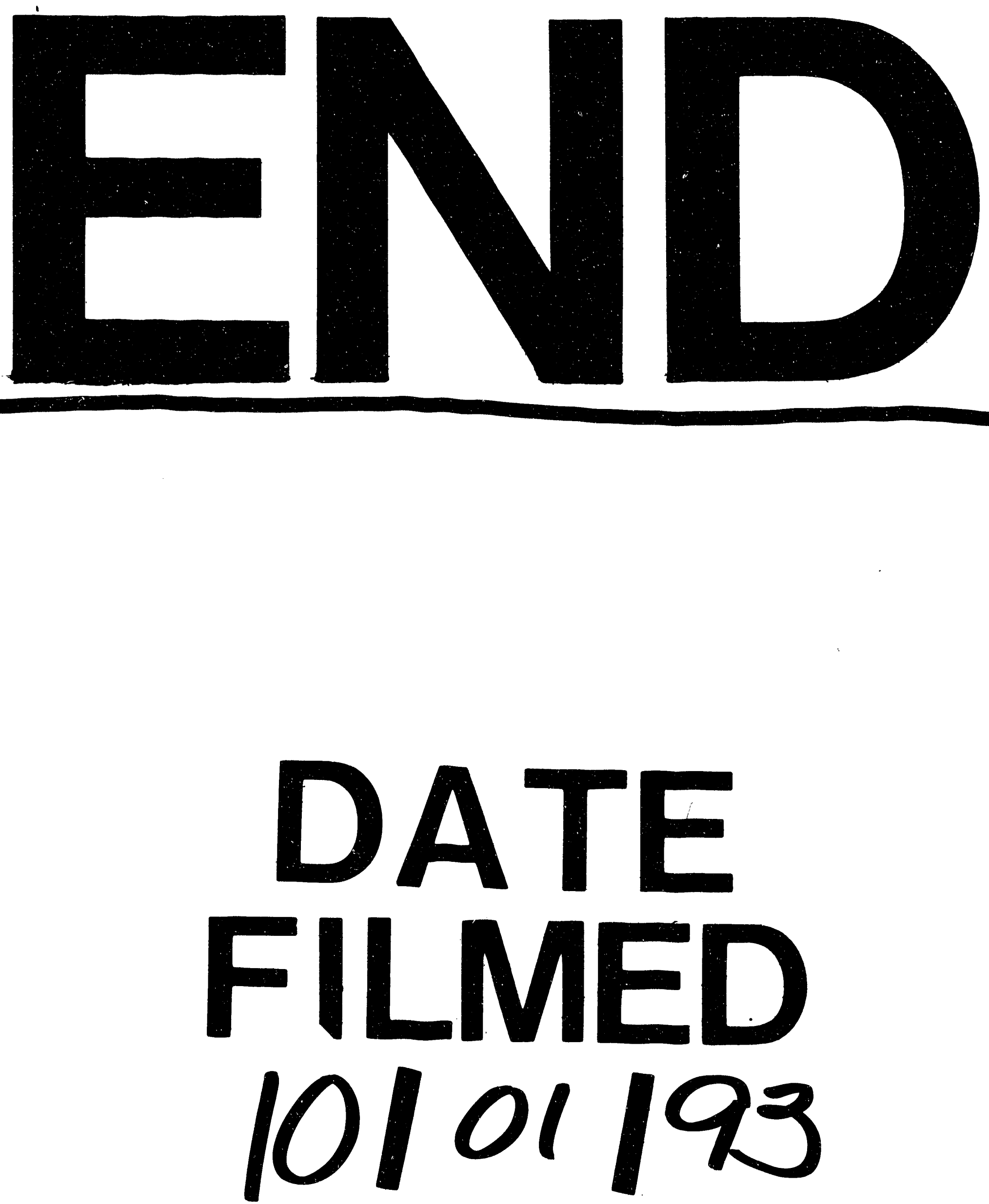

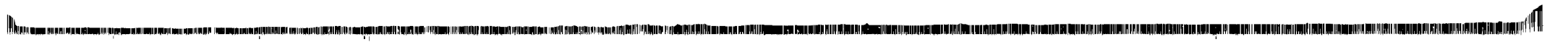




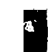

